\title{
Transitioning to Online Teaching in An Undergraduate APPliEd ENGINEERING COURSE
}

\author{
Pedram Mortazavi ${ }^{1}$, Chiyun Zhong ${ }^{1}$, Constantin Christopoulos ${ }^{2}$ \\ ${ }^{1} \mathrm{PhD}$ Candidate, Department of Civil and Mineral Engineering, Univ. of Toronto, Toronto, ON, Canada M5S $1 \mathrm{~A} 4$. \\ ${ }^{2}$ Professor and Canada Research Chair in Seismic Resilience of Infrastructure, Dept. of Civil and Mineral Engineering, \\ Univ. of Toronto, Toronto, ON, Canada M5S 1A4. \\ pedram.mortazavi@mail.utoronto.ca,myron.zhong@mail.utoronto.ca,c.christopoulos@utoronto.ca
}

\begin{abstract}
The online teaching framework presented in this paper was developed for a third-year engineering design course at the University of Toronto, with more than 100 students located in 8 different time zones. The study was inspired by the pressing challenge faced by educators around the world in 2020 to fully transition to online teaching in a limited time, due to the COVID-19 pandemic. Different facets of the course were restructured to enhance the learning experience of students, overcome the challenges associated with online instruction, and implement active learning techniques in the course whenever possible. This initiative included the development of interactive course notes and a course map, with links to videos, $3 D$ models, asynchronous lectures, and short video explanations. Several online paltforms were used in the framework, which are discussed in detail. The proposed framework provided an alternative to achieve the desired teaching outcomes in an online teaching environment for an engineering design course which had always been taught in-person prior to the year 2020. Despite the inherent challenges of this transition to online teaching, this format allowed for active learning activities in the course, promoting learner-centered teaching, and strengthening the students' collaborative skills. Elements of the framework and the methodology outlined in this paper could be useful for other engineering courses attempting an effective transition to online instruction.
\end{abstract}

Keywords: Engineering Education, Online Teaching, Engineering, Online Instruction, COVID-19, Pandemic, Teaching Framework, Active Learning, Quercus

\section{INTRODUCTION}

In 2020, educators worldwide faced an unprecedented challenge. All classes, workshops, presentations, seminars, and teaching had to fully transition to online delivery formats. Even though technological capabilities have never been more accommodating for online teaching, transitioning from a traditional in-person engineering course to a fully online course while maintaining the students' learning experience was a daunting challenge, especially since it had to be achieved in a limited amount of time and with limited resources.

Previous studies [1] have shown that several aspects could affect the student learning experience including student-instructor interactions, students peer interactions, and the incorporation of effective teaching methods such as active learning, among others. In fact, in more classical inperson instruction, students who have a higher quality interaction with instructors tend to have higher grade-point averages and not only are more likely to successfully finish their study program with intellectual and personal growth, but also to choose a career path related to their field of study $[1,2]$. The same has been shown to be true for online courses. Studies have indicated that students' satisfaction with online courses is highly dependent on the quality of studentinstructor interactions [1, 3-4]. The importance of students' peer interactions goes even beyond that of the studentinstructor interactions. In fact, the quality of student peer interactions is recognized as the single most important aspect that affects the growth of students in undergraduate studies and their likelihood of finishing the program successfully $[1,4]$. Based on the experience of the teaching staff for this course and the interactions with students, both student-instructor and student-peer interactions are more likely to be successful in face-to-face in-person teaching. This perception is also confirmed in students' response to the questionnaire in Section 5.2. Moreover, the implementation of active learning methods, which have been shown to positively influence the students' learning experience is arguably the most difficult teaching strategy to implement in online courses [1].

In addition to these challenges with online teaching in general, the hands-on nature of the engineering profession often necessitates in-class demonstrations and the use of laboratories as part of the students' learning experience. As such, there has been a general reluctance and resistance from both the teaching community and from students to choose online teaching over in-person classrooms, especially for applied STEM courses.

The final reason that usually prevents educators from adopting online teaching, or having a successful transition to online teaching, is that even though there have been significant breakthroughs both in terms of technological advancements and in online education research, it still requires significant time and effort to familiarize oneself with online teaching tools and transition a course from the classical in-person format to an online format. The required 


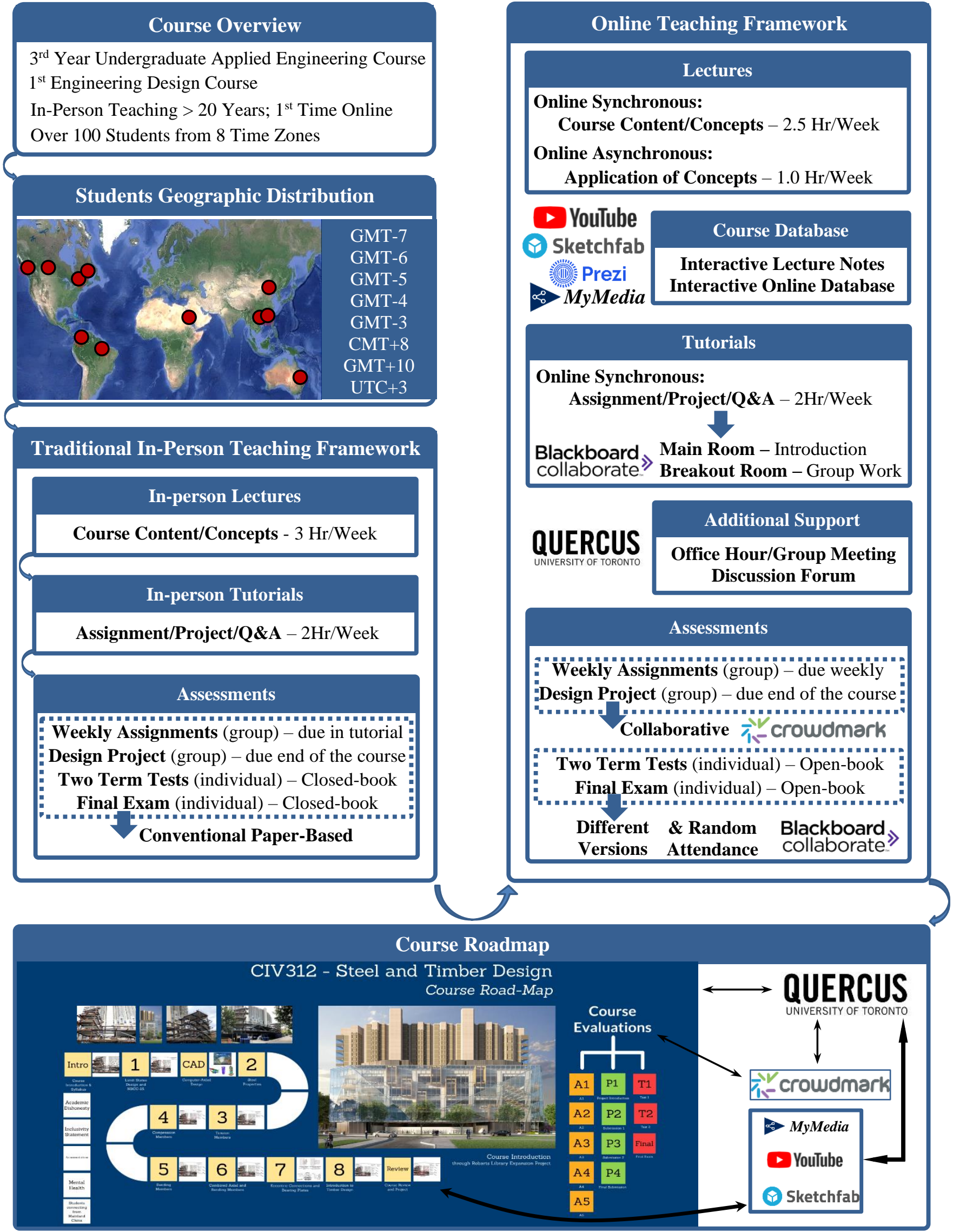

Fig. 1. Overview of the proposed framework 
Proceedings 2021 Canadian Engineering Education Association (CEEA-ACEG21) Conference

resources present a significant challenge to educators especially in a research university type environment where faculty time is split between teaching several classes, research, supervision of graduate students, broader community service and administrative duties.

This paper presents an online teaching framework that was used for restructuring a conventional engineering design course into an online one. The primary goals in the development of this framework were to: (1) maintain or enhance the learning experience of students compared to previous years, (2) maintain the quality of studentinstructor and students' peer interactions, and (3) promote and implement active learning as much as possible. The proposed framework was adopted for a third-year applied engineering course (CIV312) at the University of Toronto, which is the first structural design course in the 4-year program curriculum and has always been taught in-person prior to the fall 2020 semester. The course had more than 100 students from eight different time zones, which presented both technological challenges and time conflicts. The course management system Quercus, which is the standard platform for courses at the University of Toronto, was used in the course. The overall framework adopted for this online course is schematically illustrated in Figure 1. Like many engineering courses, CIV312 consists of four main facets, namely (1) lectures, (2) support sessions, (3) course project, and (4) assessments. In addition, a concept map was developed for the course. Each facet of the proposed teaching framework is discussed in the following sections in terms of their transition to an online format and the challenges that were faced. Lessons learned and recommendations for future applications are also presented.

\section{COURSE CONCEPT MAP}

A course map was created as a graphical depiction of the course structure, organization and topics covered in the course. The program Prezi [5] was used to develop this overall course roadmap, which can be viewed here. The course map included hyperlinks to all recorded synchronous and asynchronous lectures, the lecture notes, the teaching interactive database developed as part of the framework, course assignments, project statement, project submission details, office hours, details regarding the term tests and final assessment, and so on. It was useful to have all the course resources and organization summarized on a single interactive platform for both the students and the teaching team. Based on the experience of the authors, students tend to get overwhelmed and confused when course resources are posted on different platforms, sent through emails, Quercus announcements, etc. Students feeling overwhelmed by the multitude of platforms that have to be used to access different course materials could eventually lose interest in using all of the available tools. The course map proved useful as a reference for students, as it contained all the course material and course organization in a single location. In addition, the course map was very helpful to the teaching team for the organization of the course material, given that many of the resources were presented in this online format for the first time. Two concept maps were developed, one for the teaching team, and one for the students. The one developed for the teaching team was completed in the summer prior to the beginning of the course while the version that was developed for the students was gradually populated as the course progressed and as the topics were covered throughout the term.

\section{LECTURES}

\subsection{Lecture Notes}

In an attempt to implement active learning in the course and promote learner-centered teaching, a set of partially prepared notes was developed for the course and improved on over the past few years. This approach was helpful as it encouraged the students in taking personalized notes, while saving lecture time as the instructor could simply complete the partially prepared notes using a tablet, thus improving the quality of the notes and allowing more time for explanations. The decision to transition to partially prepared notes was validated the previous year, in a survey where the students were asked what method of lecturing provided them with the best learning experience among: i) the use of a tablet with partially prepared notes, ii) slides, iii) conventional chalk and talk (C\&T), or iv) whether this made no difference to them (No Diff).

The result of the survey is provided in Figure 2 and, as can be observed, the majority of students chose the use of a tablet with partially prepared notes as the preferable method of course content delivery. It is hypothesized that the majority of the students preferred the use of the Tablet because it resembles the classical chalk and talk method, while making it easier for students to take personalized notes, follow the explanations, and have a high-quality set of notes for studying. When students were asked about the speed of the lecture using a tablet with partially prepared notes, the results were favorable as shown in Figure 3.

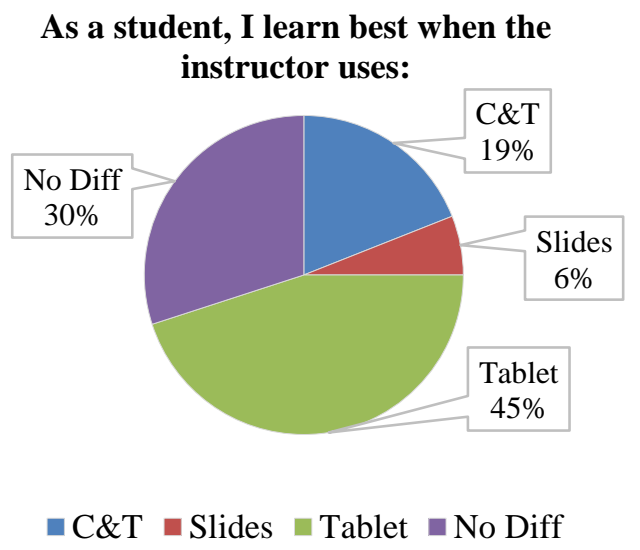

Fig. 2. Results of student survey in Fall 2019 (105 students) 
The speed of lecture material in this course is:

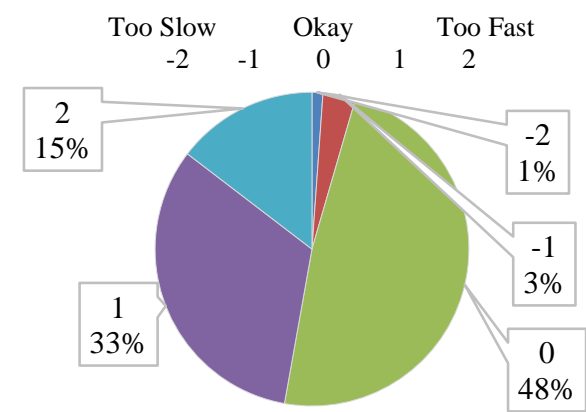

$$
\square-2 \square-1 \square 0 \square 1 \square 2
$$

Fig. 3. Results of student survey in Fall 2019 (105 students)

As part of the transition to an online format, the decision was made to further improve the partially prepared lecture notes into interactive course notes which included hyperlinks to a database of 3 dimensional (3D) interactive models, pre-recorded problem solving asynchronous lectures, short explanation videos, and course concept videos including conceptual animations, videos of educational laboratory tests, and videos demonstrating key concepts in the course. This was deemed a necessary and important step as many topics in engineering require inperson demonstrations through teaching props or laboratory tests; neither of which are available in an online format. The decision to create the online database that is discussed in the next section and its inclusion in the course concept map and the course notes was done to make up for that. Each of these aspects which were used in the interactive course notes is explained in the following section along with the platform that was used to deliver each of them.

\subsection{Online Database}

The 3D interactive models were developed using Solidworks [6] and uploaded to a Sketchfab [7] account made for CIV312. Using Sketchfab, links to these drawings were generated, which were then included in the interactive partially prepared course notes as hyperlinks. Examples of this can be found here, for a steel column base plate and concrete foundation detail, and here for a four-story steel structure. The use of 3D illustrations proved useful during lecture times, tutorials, and office hours for reinforcing the students' understanding of the course material. A total of fifteen 3D models were developed for different sections of the course.

Prerecorded problem-solving videos were made for different topics in the course, which were primarily used as asynchronous lectures, as further discussed below.

Short explanation videos and video announcements were also developed in the online database. An effort was made to keep the length of such videos between six to ten minutes, or less for maximum effectiveness [8,1].

Conceptual animations and videos of laboratory tests were either gathered from an available database of online videos or generated where required. Such videos and simulations allowed students to visualize the physical concepts that are taught in the lectures. The online format allowed to include significantly more experiments and demonstrations as the time and effort required to carry out all of these experiments in person would be prohibitive. In addition, such videos provide an excellent opportunity to promote active learning in the classroom by asking teams of students to predict the behavior prior to showing the videos. An example of this, showing the failure of a timber member with and without sheathing can be found here. Another example that shows the mechanics of an eccentrically loaded bolted connection can be found here.

A total of 79 videos, consisting of 22 asynchronous lectures and 57 concept videos and animations were uploaded to the course YouTube [9] account and replicated on a MyMedia [10] account which is an in-house standard video-sharing platform used at the University of Toronto. It was decided to use both platforms because while most students tend to be more comfortable and familiar with YouTube, this platform is not accessible in some countries. Therefore, MyMedia was used as a secondary platform for all videos.

\subsection{Synchronous and Asynchronous Lectures}

An important decision had to be made on the structure and allocated times for lectures vs tutorials, theory vs examples, and synchronous vs asynchronous formats. Given the geographic location of students, asynchronous lectures tend to give students the freedom to follow the lectures at reasonable hours in their respective time zones and manage their time more effectively. On the other hand, synchronous lectures are generally preferable for conceptual topics as they allow for in-person interactions with the instructor. It was decided to deliver two of the three-hour weekly lectures in a synchronous format covering the course theory and preliminary examples. The third weekly lecture hour was delivered in an asynchronous format and focused mostly on fully solved examples. This division between theory and examples was informed by the results of student surveys in previous years. As can be observed in Figure 4 and 5, and as expected for an applied engineering design course, most students in the course tend to feel more comfortable with the course content if more time is spent on examples.

\section{I would learn better if more time was} spent on examples

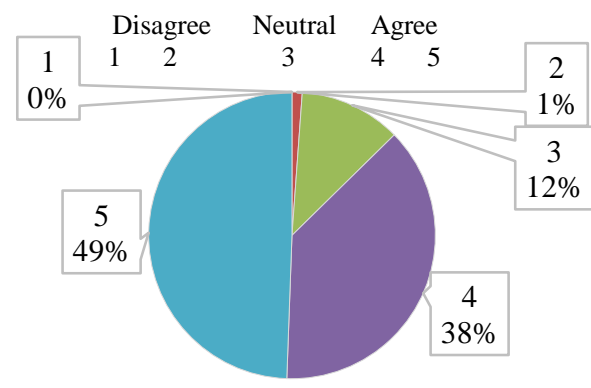

$$
\square 1 \square 2 \square 3 \square 4 \square 5
$$

Fig. 4. Results of student survey in Fall 2019 (105 students) 
I would learn better if more time was spent on theory

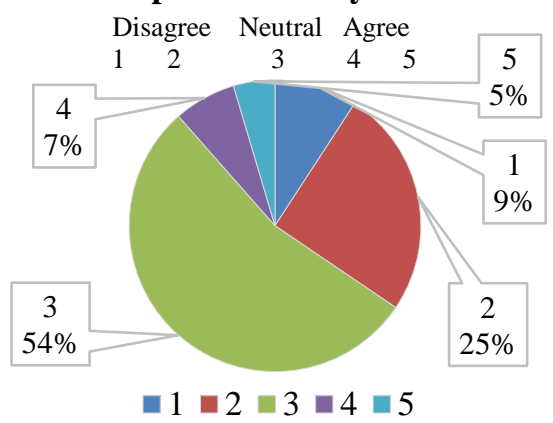

Fig. 5. Results of student survey in Fall 2019 (105 students)

\section{ADDITIONAL COURSE SUPPORT}

Similar to other facets of the proposed framework, the online teaching support elements presented in this section were designed to emulate the teaching support elements that are typically offered in conventional in-person learning experiences. Feedback from both the students (discussed in details in Section 5) and the teaching assistants (TA) confirmed that a similar level of interaction was achieved when compared to conventional in-person learning experiences.

\subsection{Tutorials}

CIV312 tutorials were usually held in person, within two large classrooms of about 50 students, with 2 to 3 TAs in each room. Each tutorial session had an opening introduction and discussion of key concepts delivered by the TAs which typically lasted between 10 to 20 minutes. Students would then work in groups of four on weekly assignments, or the course project, and TAs would circulate around to answer their questions. This approach was observed over the years to promote collaborative work and learner-centered teaching.

To retain the collaborative learning environment of the tutorials, online tutorials were organized as synchronous sessions through BB Collaborate (or using other general software/platform with similar capabilities). Each session would start in the Main Room with the opening introduction and discussion of key concepts similar to the in-person tutorials. Group work sessions were then emulated by dividing students into Breakout Rooms within the Main Room, such that students within the same group had a virtual study room for them to discuss, share screen, video, etc., while TAs and the Instructor continued to circulate between groups (i.e. Breakout Room) to answer questions, all of which were done without interrupting other groups. On average each TA would spend $10-15$ minutes per group, visiting $10-12$ groups, in each tutorial. Having 5 TAs in each tutorial resulted in each group having at least three TA interactions, for a total of around $30-45$ minutes of contact time. The course instructor was also present in the main room and would visit groups upon request as a secondary resource and for immediate support in addition to being available for students to come into the main room to ask further questions. The instructor was also CEEA-ACEG21; Paper 008

UPEI; June $20-23,2021$ in contact with the TAs through a separate chat to answer TA questions and ensure consistency in the interactions of all TAs with the students. The structure of the tutorials in the opening lecture and breakout room session is conceptually illustrated in Figure 6.

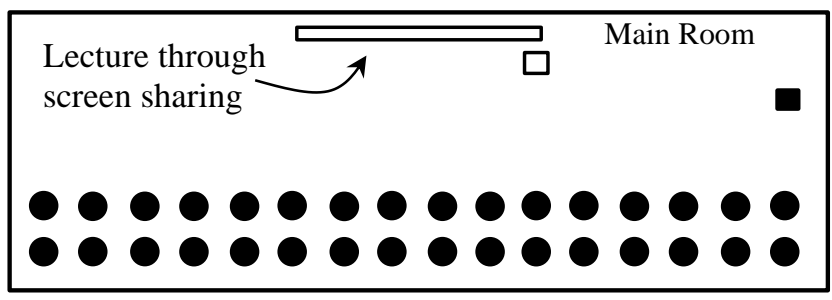

(a)

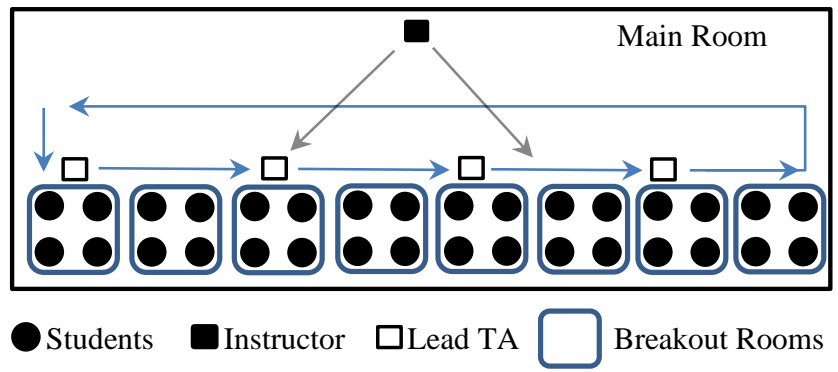

(b)

Fig. 6. Structure of virtual tutorials (a) opening segment, and (b) groupwork session

\subsection{Office hours}

Office hours were held using BB-Collaborate in a manner similar to the tutorials discussed above. Conventional office hours typically are delivered in one of the following forms: (1) an open discussion between a student and the TA where the other students listen and learn from the general discussion, or (2) groups of students interacting more exclusively with a TA, while the other students wait for their turn to ask their questions. The first form of office hours allows the students to listen to discussions and follow the answer to questions that they may not have originally thought of. The second form has the advantage of allowing students to manage their time effectively and attend the office hour as long as they deem it necessary.

It was decided to have office hours that would simultaneously adopt both of the above-mentioned formats, using the following approach. During office hours, a lead TA hosted the Main Room while leading the general discussion in this room. Students who believed they could benefit from the ongoing publicly-answered questions and discussions were encouraged to remain in the same room and took turns asking questions. Students who preferred to get answers to their questions privately and leave the office hour afterwards were assigned to one of the Breakout Rooms hosted by other TAs. The format of the TA office hours is conceptually shown in Figure 7. For any questions that were not covered during the office hours or the tutorials, students were asked to post them on a discussion forum, which is discussed in the next section.

In addition to this, the instructor held office hours after each synchronous lecture. The office hours often lasted for 15-20 minutes and were recorded so that the students could 
Proceedings 2021 Canadian Engineering Education Association (CEEA-ACEG21) Conference

access them after the lectures and throughout the term. This enriched the students' experience as they did not have to wait for the regular office hours for asking questions on the topics covered in the lecture.

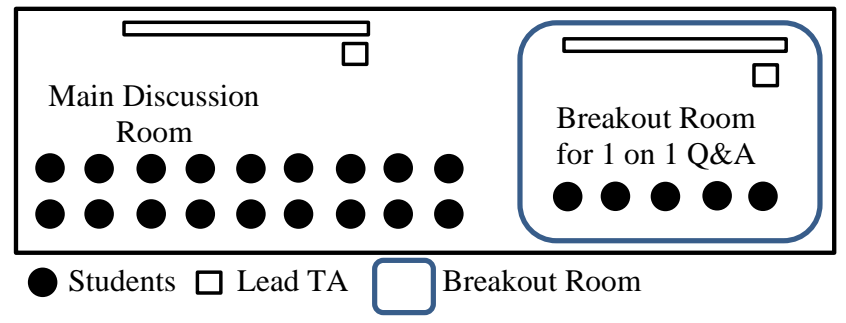

Fig. 7. Structure of virtual TA office hours

\subsection{Discussion Forums}

The discussion forums served as a centralized database for all teaching support offered in this course and were organized on Quercus in two main sections for each chapter of the course: 1) Common Q\&A section; 2) Interactive discussion forums. The common Q\&A sections were created to organize and post all common questions gathered from each tutorial and office hours, as well as from the feedback from the instructor after each synchronous lecture session, and the corresponding detailed answers. The interactive threaded forum was used for students to post their questions, while everyone in the class was encouraged to have discussions under each question and provide their thoughts. The TAs checked the discussion forum several times a day to provide clarifications and corrections if necessary. The main advantages of discussion forums are twofold: (1) they encourage students to participate in discussions and in helping their classmates, while reinforcing their own learning (learner-centered teaching and a form of the flipped classroom); and (2) it serves as an online database or reference for questions that can be accessed at any time, such that students review the discussion forum first before trying to reach out to the teaching staff, which requires fewer resources from the teaching team.

\section{ASSESSMENT}

Recognizing the restrictions and challenges associated with online assessments, the methods discussed in this section were primarily designed with two purposes: (1) to ensure academic integrity in assessments in an online course as much as it was possible without the need to drastically redesign the assessment methods, thus trying to ensure a smooth transition from in-person to online assessments, and (2) to simplify the online marking process, thus enabling the teaching team to provide more timely feedback to students.

\subsection{Assignments and Projects}

Posting, submission, and grading of assignments and the course project were both done through Crowdmark [11], a grading platform, which facilitates the grading of large numbers of submissions by multiple graders (i.e. TAs). Based on the experience in this course, grading in Crowdmark generally reduced the marking time, compared CEEA-ACEG21; Paper 008

UPEI; June 20 - 23, 2021

$$
-6 \text { of } 8-
$$

to previous years. This not only allows TAs to provide feedback to students in a timely manner, but also allows the teaching team to allocate resources to other tasks. Crowdmark also provides insightful data visualizations of student performance on each question which the instructor and TAs can use to provide more targeted teaching.

\subsection{Term Tests and Final Assessment}

The importance of maintaining academic integrity both throughout the course and during the final assessment cannot be overstated, along with the challenges of achieving this in an online format. In fact, this has been the single most important criterion that has affected the structure of paperbased in-person assessments in conventional instruction. For in-person assessments, students must appear for their exams in person on a designated day and time. They are only allowed to bring the resources that the course instructor has specified and must bring their valid student cards. Several invigilators are often required to supervise the students throughout the exam, ensure that order is maintained during the session, and check the students' attendance and identification. By considering measures that are taken in conventional in-person examinations, it is clear that conducting online student assessment presents challenges when it comes to maintaining academic integrity, and therefore, requires careful planning.

For the midterms and the final assessment, conventional paper-based evaluations were mostly retained in the course with some adjustments to better fit the online-format while making best efforts to maintain academic integrity. Four different versions of the tests were developed, and they were assigned such that each student in the same study group would receive a different test. This was also done for the final summative assessments. Questions were made available to the students at the beginning of the exam and BB-Collaborate sessions were used to mimic the traditional exam invigilation by hosting each test in the main room of a BB Collaborate session, and randomly using designated Breakout Rooms for an identification check. The students were asked to attend the midterms and final assessments with access to the internet, a webcam, and their student identification. In addition, it was decided to have an open book examination. An additional Breakout Room allowed students to ask their questions from the course instructor without interrupting others. A schematic illustration of the virtual sessions for term tests and final assessments is shown in Figure 8.

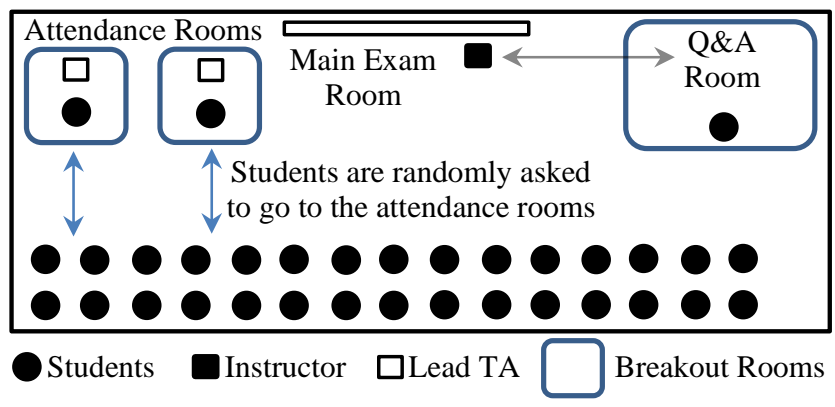

Fig. 8. Structure of virtual assessment sessions

The students were simply asked to be present in the BB 
Proceedings 2021 Canadian Engineering Education Association (CEEA-ACEG21) Conference

Collaborate session, provide identification to the TAs in the

Breakout Room upon request by sharing their camera, and

to scan and upload their answers at the end of the assessment. For the latter, the students were provided with 15 minutes of additional time for this.

The second important criterion in planning for the midterms and final assessments were to simplify the marking process. For short answer questions with a definitive correct answer, the course management tool, Quercus, was used. This was deemed more efficient for the marking of such questions as marking could be done automatically with predefined correct answers. However, long answer questions were provided to the students in a paper-based format, given that conventional paper-based evaluations are more suitable for engineering design courses that involve a substantial amount of calculations.

For marking the student assessment submissions, the instructor could choose either to print out the submissions for marking, or to mark through an online platform such as Crowdmark, instead of being limited to the specific format of an online assessment platform.

\section{FOCUS GROUP FEEDBACK}

To gather some feedback on the adopted framework, a focus group of 10 students in the course was formed a few weeks after the end of the semester. The students were selected based on their level of involvement with all activities in the course, including attending synchronous lectures, actively participating during tutorials, asking questions during office hours. The students were asked to provide feedback on different aspects of the course through 11 questions accompanied by some comments. The questions are listed below, and the results of the survey are provided in Figure 9.

\subsection{Questionnaire}

1. As a student experiencing online education for the first time, I was apprehensive at the start of the term.

2. I actively attended all lectures and all tutorials.

3. The course map was a useful resource to understand and navigate the course material and resources.

4. I found that the course had a good balance in terms of time spent between theory and examples.

5. I found that the course had a good balance in terms of time spent between synchronous and asynchronous lectures.

6. The online database of 3D models, animations, videos, lab tests, recorded examples, etc. helped me better understand course concepts.

7. I found that using the interactive partially prepared notes enhanced my learning experience.

8. I found that the discussion board was a useful resource, which at times removed the need for me to contact the course instructors/TAs for questions.

9. My experience of office hours emulated my experience with real in person office hours.

10. Overall, I found this online course stimulating

11. After this experience, I find myself more open to taking online courses.

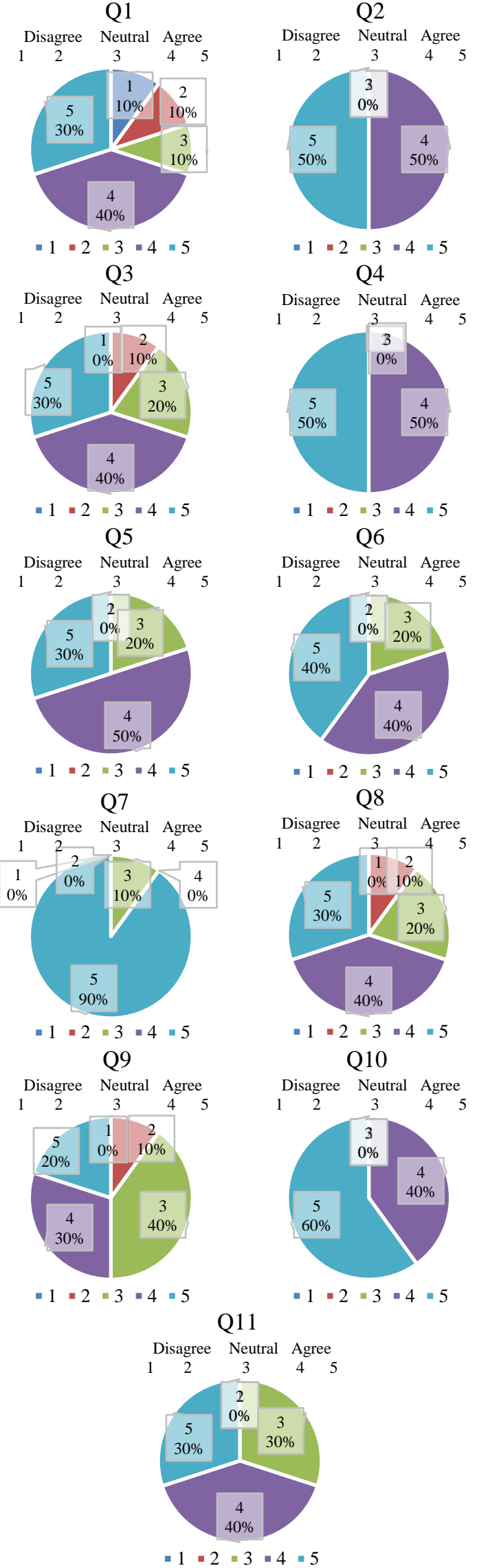

Fig. 9. Results of the focus group questionnaire (10 students) 
Proceedings 2021 Canadian Engineering Education Association (CEEA-ACEG21) Conference

\subsection{Discussion}

The results of the focus group questionnaire are first discussed considering the entirety of the adopted framework. Next, the results are discussed in more detail while also considering the different facets of the framework. It is worth noting that these results represent students who actively participated in all aspects of the course, not the entire class. As shown in the results from question 2, 10 out of 10 students in this focus group actively participated in the course.

The results indicate that 7 out of 10 students were anxious at the beginning of the term about taking online courses, with one student being neutral. However, at the conclusion of the semester, 10 out of 10 students found the course to be stimulating and 7 out of 10 students expressed that they would be more open to taking online courses in the future. This indicates an overall positive outcome for the proposed framework.

Seven out of 10 students found the course map useful in navigating the course and understanding the course concepts and how they fit in the overall structure of the course. It must also be noted that the use of the map is not common, and many students experienced this for the first time. It is likely that some students did not feel comfortable with the concept map for this reason. The course instructor actively used the map at the beginning of each lecture to help make the students more familiar with this approach.

All students believed that the course had a good balance between theory and examples and 8 out of 10 students were satisfied with the balance between synchronous and asynchronous lectures.

Eight out of 10 students found that the online database, which was developed and used in the proposed framework, enhanced their learning experience. Nine out of 10 students found that the partially prepared interactive notes were helpful to their learning.

While the majority of the students (7 out of 10) found the discussion boards useful 2 students felt neutral about this and one student disagreed. This seems to justify the use of this approach, especially considering this also frees up significant resources, and more importantly, promotes a learner-centered teaching environment.

Half the students felt that the office hours emulated their experience of real office hours, while 4 students felt neutral about this.

\section{CONCLUDING REMARKS}

This paper presented a framework that could be useful for STEM courses transitioning from conventional inperson face-to-face instruction to fully online courses with reasonable time and resources, while maintaining key elements of the quality of students' learning experience compared to in-person learning. As part of the proposed framework, a set of interactive lecture notes with an interactive online database were developed. The framework was successfully used for a third-year engineering design course at the University of Toronto in the fall of 2020 when all classes had to be transitioned to online delivery. This experience provided insights that CEEA-ACEG21; Paper 008 UPEI; June $20-23,2021$ could be useful for other applied STEM courses whether it is for fully online instruction or for hybrid online and inperson instruction.

The study leads to the following conclusions:

- Although significant advancements have been made in online teaching in recent years, fully transitioning to online teaching while maintaining the quality of the students' learning experience remains a challenge, requiring considerable resources to achieve.

- In discussion with students, the greatest challenge they faced during the 2020 COVID-19 pandemic with online classes was the variety of instruction platforms that educators used in each class. Therefore, more consistent use of teaching platforms across departments and faculties is desirable.

- The proposed framework was deemed to have been effective in enhancing the learning experience of students as well as the teaching experience of the teaching team. The result of the focus group questionnaire, albeit with a limited group size, shows the effectiveness of the proposed framework.

- Grading through Crowdmark proved to be efficient for long answer questions, while Quercus proved to be a more efficient platform for short answer questions. Using both platforms allowed the course TAs to allocate their resources to other tasks in the course.

- Aspects and newly-available technologies presented in this work can be considered as potential tools to enhance in-person teaching, even after the COVID19 pandemic.

\section{References}

[1] Richard M. Felder and Rebecca Brent, Teaching and learning STEM: A practical guide. San Francisco, CA: Jossey-Bass, 2016. \{ISBN: 9781118925812$\}$

[2] Alexander W. Astin, What Matters in College: Four critical years revisited. San Francisco, CA: Jossey-Bass, 1993. \{ISBN: 1-55542-492-9\}

[3] Judith Boettcher and Rita-Marie Conrad, The online teaching survival guide: Simple and practical pedagogical tips. San Francisco, CA: Jossey-Bass, 2010. \{ISBN: 98040423530\}

[4] Rebecca Croxton, "The role of interactivity in student satisfaction and persistence in online learning," Journal of Online Learning and Teaching, vol. 10, no. 2, pp. 314325, 2014.

[5] https://prezi.com/

[6] https://www.solidworks.com/

[7] www.sketchfab.com/

[8] Philip J. Guo, Juho Kim, and Rob Rubin, "How video production affects student engagement: an empirical study of MOOC videos" Proceedings of the first ACM Conference on Learning @ scale conference, pp 41-50, 2014.

[9] https://www.youtube.com/

[10] https://play.library.utoronto.ca/

[11] https://crowdmark.com/ 